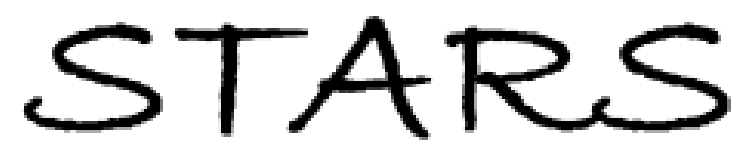

University of Central Florida

STARS

$1-1-2006$

\title{
Self-calibrating hybrid wavelength, polarization, and time- multiplexed heterodyne interferometers for Angstrom precision measurements
}

\author{
Nabeel A. Riza \\ University of Central Florida \\ Muzammil A. Arain \\ University of Central Florida \\ Farzan N. Ghauri \\ University of Central Florida
}

Find similar works at: https://stars.library.ucf.edu/facultybib2000

University of Central Florida Libraries http://library.ucf.edu

This Article; Proceedings Paper is brought to you for free and open access by the Faculty Bibliography at STARS. It has been accepted for inclusion in Faculty Bibliography 2000s by an authorized administrator of STARS. For more information, please contact STARS@ucf.edu.

\section{Recommended Citation}

Riza, Nabeel A.; Arain, Muzammil A.; and Ghauri, Farzan N., "Self-calibrating hybrid wavelength, polarization, and time-multiplexed heterodyne interferometers for Angstrom precision measurements" (2006). Faculty Bibliography 2000s. 6510.

https://stars.library.ucf.edu/facultybib2000/6510

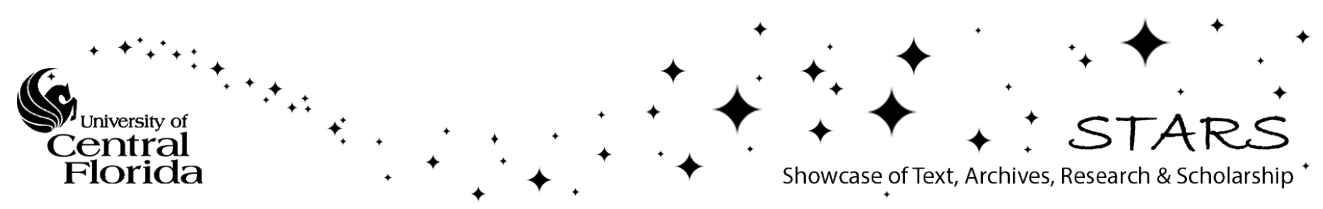




\section{Self-calibrating hybrid wavelength, polarization, and time-multiplexed heterodyne interferometers for Angstrom precision measurements}

\author{
Nabeel A. Riza, FELLOW SPIE \\ Muzammil A. Arain \\ Farzan N. Ghauri \\ University of Central Florida \\ Photonics Information Processing \\ Laboratory \\ College of Optics and Photonics \\ Center for Research and Education \\ in Optics and Lasers \\ 4000 Central Florida Boulevard \\ Orlando, Florida 32816-2700 \\ E-mail: riza@creol.ucf.edu
}

\begin{abstract}
Propose here unique wavelength and hybrid wavelengthpolarization and wavelength-polarization-time-multiplexed heterodyne optical interferometers using an internal self-referencing scheme enabling Angstrom level sensitivity optical path-length measurements. As a first step, a proof-of-concept wavelength multiplexed scanning heterodyne interferometer is built to test the surface quality of a test mirror, demonstrating $\pm 100 \AA$ profile variations with a $0.9 \AA$ accuracy. The hybrid wavelength-polarization and wavelength-polarization-timemultiplexed interferometers can be used to form spectrally coded distributed sensors. () 2006 Society of Photo-Optical Instrumentation Engineers.
\end{abstract} [DOI: $10.1117 / 1.2404680]$

Subject terms: acousto-optic deflectors; heterodyne optical interferometers; optical sensors; scanning interferometers.

Paper 060072R received Jan. 27, 2006; revised manuscript received May 7, 2006; accepted for publication May 11, 2006; published online Dec. 19, 2006. This paper is a revision of a paper presented at the SPIE conference on Enabling Photonics Technologies for Defense, Security and Aerospace Applications, Orlando, Florida, Mar. 2005. The paper presented there appears (unrefereed) in SPIE proceedings Vol. 5814.

\section{Introduction}

Optical interferometry is a useful tool for a host of applications that include optical surface characterization, optical sensors, optical path-length (OPL) measurements, and displacement measurements. A number of interferometer configurations have been proposed for different applications. ${ }^{1-5}$ Heterodyne interferometers, in particular, are suitable for high-precision displacement or surface roughness measurements in high-noise environments. Specifically, heterodyne common path interferometers are especially tolerant to mechanical vibrations and environmental fluctuations, making them suitable for high-precision measurements. Another desirable feature for any interferometer is a no-moving parts scanning capability. A number of scanning heterodyne interferometers have been proposed, including inline acousto-optic interferometers with high-speed scanning features. ${ }^{6,7}$ In general, using heterodyne interferometry, for instance, in precision optical path-length measurements, requires a stable radio-frequency $(\mathrm{RF})$ reference signal for $\mathrm{RF}$ phase comparison using an RF phase meter. One method to achieve this goal is comparing the output RF signal from the heterodyne interferometer with the stable external RF source that optically modulates the interferometer. Nevertheless, using this external RF signal from the RF signal generator makes the OPL measurements sensitive to the changing environmental conditions that only affect the RF signal produced by the interferometer. ${ }^{8}$ A preferred solution is to use an internally generated reference RF signal pro-

0091-3286/2006/\$22.00 @ 2006 SPIE duced via the interferometer optics and RF electronics, thus generating an RF reference signal whose noise is correlated to the environmental conditions. Because the OPL signal $\mathrm{RF}$ is also produced by the same interferometer optics and electronics, the signal and reference RFs now have correlated noise, a condition to maximize phase detection signalto-noise ratio (SNR) in the RF phase meter.

Recently, a polarization-multiplexed interferometer design was designed and demonstrated to enable internal generation of the reference RF. ${ }^{9,10}$ In this paper, an alternate technique based on wavelength multiplexing within the earlier proposed acousto-optic heterodyne interferometer ${ }^{6}$ is used to enable the desired internal RF reference signal generation. Specifically, the wavelength-multiplexed interferometer uses two different wavelengths to provide the stable self-calibrating reference signal along with the signal RF. The proposed interferometer uses a single acousto-optic deflector (AOD) or Bragg cell in a double-diffraction reflection geometry to realize a compact OPL measurement instrument that can electronically scan the test zone in one dimension. A proof-of-concept wavelength-multiplexed interferometer is built and tested using a test plate for surface profile measurements. An extension of the proposed interferometer using both wavelength and polarization to form a hybrid design is shown that can work as a distributed interferometric optical sensor for instantaneous measurements. A further simplified hybrid wavelength-polarization-timemultiplexed architecture with minimal hardware is proposed, where noninstantaneous sensing is adequate. The rest of the paper describes the details of the proposed interferometers. 


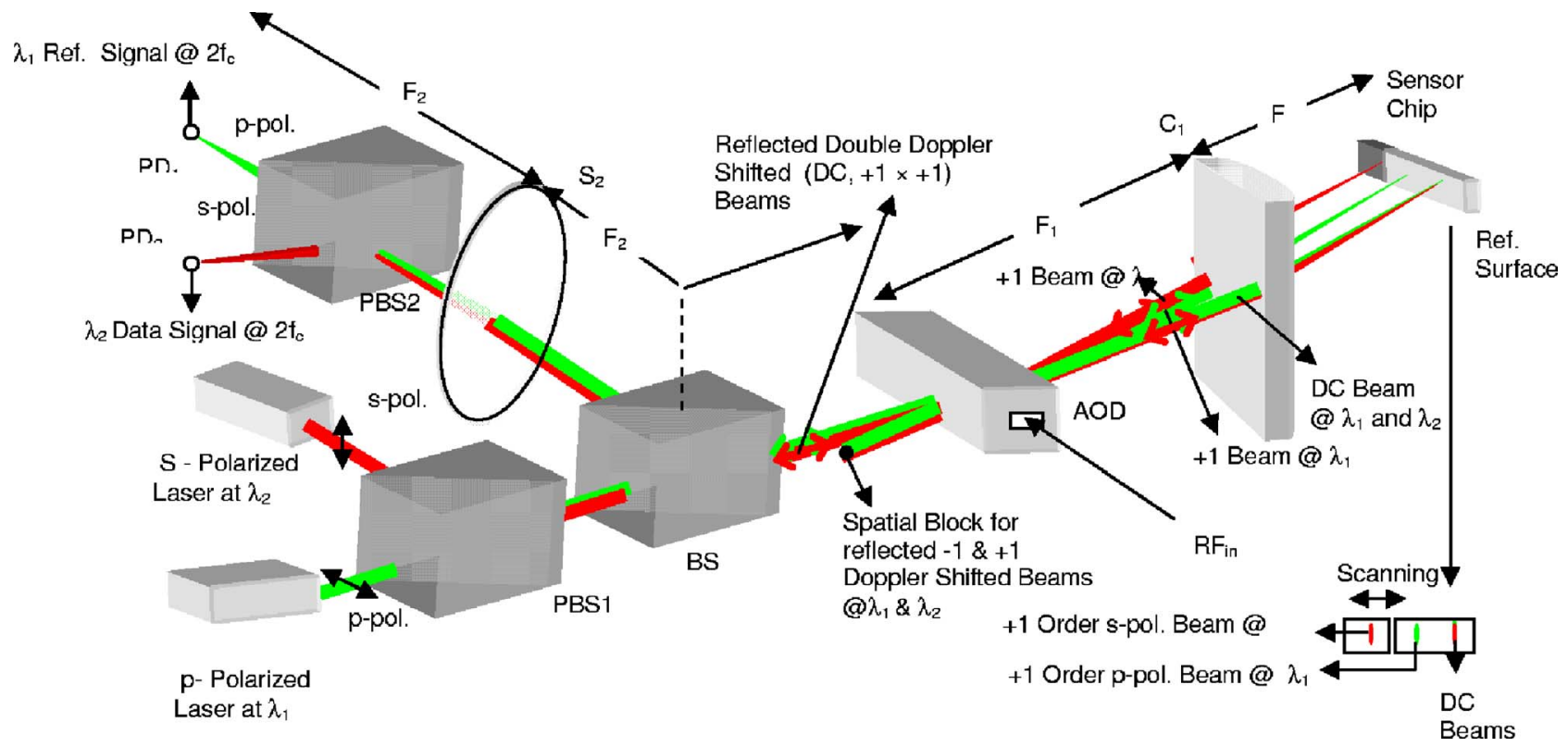

Fig. 1 Proposed wavelength multiplexed acousto-optic scanning heterodyne interferometer with RF self-referencing capability. BS: beamsplitter; PBS: polarization beamsplitter; AOD: acousto-optic deflector; PD: photodetector.

\section{Acousto-Optic Self-Referencing Wavelength-Multiplexed Heterodyne Interferometer Design}

The proposed architecture of the wavelength-multiplexed heterodyne interferometer is shown in Fig. $1 .{ }^{11}$ Here two independent linearly polarized collimated laser beams of wavelengths $\lambda_{1}$ (for reference RF generation) and $\lambda_{2}$ (for OPL signal RF generation) with orthogonal polarizations (p: horizontal and s: vertical) are used as optical sources. A cube polarization beamsplitter (PBS1) combines the two $p$ and $\mathrm{s}$ polarization beams, making them inline. Next, the two beams pass through a cube 50:50 beamsplitter (BS) to be incident at a central Bragg angle (i.e., between the Bragg angles for the two wavelengths) on the AOD that is fed by an RF of $f_{c}$. Note that here a broadband operation AOD is required that ensures sufficient diffraction efficiency for the two wavelengths. The choice of wavelengths and laser powers, AOD RF drive power and frequency, AOD material type and design, photo-detection optics, and related RF amplifiers can be optimized to the specific application requirements based on the sensor test sample. In other words, the relatively fixed-power reference optical/RF signal can be predesigned such that the signal RF delivers the best SNR for the samples to be tested by the instrument.

The isotropic diffraction Bragg cell incident inline beams produces two +1 diffracted order $\mathrm{p}$ (for $\lambda_{1}$ ) and $\mathrm{s}$ (for $\lambda_{2}$ ) polarized beams that are angularly separated on account of the different Bragg deflection angles produced from the AOD corresponding to the two different wavelengths but same AOD RF drive frequency $f_{c}$. These +1 -order beams and the two inline DC beams pass through an $F_{1}$ focal length cylindrical lens $C_{1}$ that makes all the beams parallel with respect to each other. The two DC beams at $\lambda_{1}$ and $\lambda_{2}$ and the +1 -order reference beam at $\lambda_{1}$ are made to retroreflect from a highly flat, larger reference mirror, while the +1 -order signal beam at $\lambda_{2}$ is directed to the test surface or reflecting material sensor chip under study and OPL measurement. All four retroreflected beams (two inline DC and two separated +1 order) travel back to the AOD to undergo Bragg diffraction. The two DC beams pass through the AOD to produce (1) two -1-order beams that are spatially separated from the original incident beams and are spatially blocked in the system and (2) two DC beams that are the undiffracted beams that are inline with the original incident beam and hence act as the reference optical beams at $\lambda_{1}$ and $\lambda_{2}$ for eventual heterodyne detection at the point photo-detectors. In addition, the two angularly separated +1 -order beams pass through the AOD again to produce (1) two +1 -order beams that are spatially separated from the original incident beam path and hence are spatially blocked in the instrument and (2) two doublediffracted $(+1 \times+1)$-order beams at $\lambda_{1}$ (for reference heterodyne detection) and $\lambda_{2}$ (for test signal heterodyne detection) that are inline with the original incident beam path. Note that the incident beams on the AOD are collimated laser beams, and the use of cylindrical lens $C_{1}$ converts these near-circular beams in focused tiny lines on the sensor chip/reference mirror plane (see Fig. 1). Nevertheless, on return to the photo-detectors, an $F_{2}$ focal length spherical lens $S_{2}$ produces focused spots on the photo-detection planes. The focal lengths of the two lenses can be adjusted to modify imaging magnification powers between the sensor chip plane and the detection planes. Also note that to produce a scanning beam on the sensor chip plane, the AOD RF can be varied that causes the +1 -order $\lambda_{2}$ wavelength $s$-polarization beam to move in one dimension, hence accessing different scan zones on the chip. Changing the RF also moves the +1 -order $\lambda_{1}$ wavelength p-polarization beam on the reference mirror; hence, the mirror size is designed to be large enough to cover this scan 
zone to enable reference beam retroreflection. The other two DC beams on the reference mirror stay stationary when the AOD RF changes. The key point to note is that after retroreflection and double Bragg diffraction, the original scanning +1 -order $\lambda_{2}$ wavelength s-polarization beam becomes the stationary $(+1 \times+1)$-order $\lambda_{2}$ wavelength s-polarization beam that is also inline with the returning DC beams for perfect spatially matched heterodyne detection at the high-speed photo-diodes. Then another cube PBS2 placed in the beams' return path is used to spatially separate the $\mathrm{p}$ and s polarized $\lambda_{1}$ and $\lambda_{2}$ beam pairs, respectively. The $\lambda_{1}$ p-polarization beam pair is incident on the $\mathrm{PD}_{1}$ high-speed photo-detector and produces the reference $\mathrm{RF}$ at $f_{c}$ given by

$i_{R}(t)=A_{R} \cos \left[4 \pi f_{c} t+\phi_{n}(t)\right]$,

where $A_{R}$ is a fixed RF amplitude term and $\phi_{n}(t)$ is the relative phase noise experienced by the $\lambda_{1}$ beam pair. Note that because of the near-inline design of the interferometer, the $\lambda_{2}$ beam pair also experiences the same relative temporal phase noise in the instrument, thus allowing phase noise cancellation in the RF phase meter. Hence, the $\lambda_{2}$ s-polarization beampair is incident on the $\mathrm{PD}_{2}$ high-speed photo-detector and produces the OPL signal/data RF at $2 f_{c}$ given by

$i_{S}(t)=A_{S} \cos \left[4 \pi f_{c} t+\theta_{S}+\phi_{n}(t)\right]$,

where $A_{S}$ and $\theta_{S}$ are the test sample-dependent RF amplitude term and phase terms, respectively. Here $\theta_{s}=\left(4 \pi / \lambda_{2}\right)$ $\times\left[n\left(\lambda_{2}\right) d_{s}\right]$, where $n\left(\lambda_{2}\right)$ is the test sample material refractive index at $\lambda_{2}$ and $d_{s}$ is the test sample thickness (or profile when considering a reflective sample) relative to the flat reference mirror. Therefore, as originally intended, the instrument internally produces two $\phi_{n}(t)$ phase noisecorrelated RF signals at the same $2 f_{c}$ frequency but with the reference $\mathrm{RF}$ from $\mathrm{PD}_{1}$ have a fixed phase and amplitude while the designated OPL data signal RF from $\mathrm{PD}_{2}$ have an RF phase and amplitude value dependent on the OPL and other material properties of the sensing chip zone. Hence, these two RF signals $i_{S}(t)$ and $i_{R}(t)$ are fed into a lock-in amplifier to electronically mix and low-pass filter the signal to produce the sensor measurement signal $i_{M}(t)$ given by

$i_{M}(t) \propto A_{S} \cos \theta_{S}$.

Note that indeed Eq. (3) gives the desired test sample OPL and optical reflection amplitude information. For example, for a highly reflective mirrored test sample, the detected RF phase $\theta_{S}$ gives the sample surface profile information. This relative RF phase shift will change as optical beam scanning in one dimension over a given test sample is done by changing the RF drive frequency of the AOD. Since the two beams are almost collinear, any transverse deviation caused by angular misalignments is experienced by both beams and does not affect relative phase-shift information in the heterodyne detected signal. More specifically, the relative phase shift that provides information about the test surface profile is due to the axial direction distance change that comes only from the sample profile

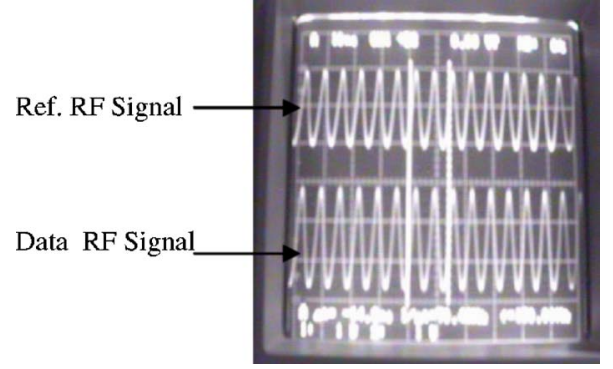

Fig. 2 Measured oscilloscope traces of the $i_{S}(t)$ and $i_{R}(t)$ RF signals using an $f_{c}=70-\mathrm{MHz}$ AOD drive signal. The upper signal is the reference signal obtained via heterodyne detection of $D C$ and $-+1 \times$ +1 )-order optical beam at wavelength $\lambda_{1}$. The lower signal is obtained via heterodyne detection of DC and $(+1 \times+1)$-order optical beam at wavelength $\lambda_{2}$.

change. As the two DC beams and the +1 -order reference beam are reflected from a flat surface of the reference mirror, these beams do not introduce any additional RF phase shift. However, the +1 -order signal beam is reflected from a different point on the surface of the test sample, leading to a possible change in relative RF phase shift in the lock-in amplifier. Hence, this measured RF phase information provides metrology data for the surface of the test sample. Using the full RF frequency range of AOD, a onedimensional surface profile map of the test sample can be generated. For a complete two-dimensional map, either the sample or the sample engaging signal beam can be translated in the orthogonal linear dimension.

\section{Wavelength-Multiplexed Acousto-Optic Scanning Interferometer Experimental Demonstration}

As a first demonstration of the proposed internally referenced interferometer concept using wavelength multiplexing, a high-flatness (e.g. super polished to $<1 \AA \mathrm{rms}$ microroughness) mirror is used as a reference surface while a poor-flatness-quality mirror is used as a test sample. Here, the reference surface is assumed to be a perfectly flat surface. However, in practice, the surface profile may have slight surface variations over different locations on the mirror leading to a fixed-hence known-phase offset for the reference signal in Eq. (1). Because this reference phase offset is known for a given flat when calibrating the instrument, one can subtract this phase information from the sample signal measurements to enable a true precision measurement of the test sample. An argon ion laser with a $\lambda_{1}$ wavelength of $514.5 \mathrm{~nm}$ is used as a reference wavelength while a $\lambda_{2}=632-\mathrm{nm}$ HeNe laser is used as the signal wavelength for internal RF reference signal generation. An AOD with an $f_{c}$ center frequency of $70 \mathrm{MHz}$ and bandwidth of $40 \mathrm{MHz}$ is used for the experiment. Fig. 2 shows the oscilloscope traces of the two detected $i_{S}(t)$ and $i_{R}(t)$ RF signals. The $\theta_{S}$ phase difference is measured using a RF lock-in amplifier SR 844 from Stanford Research Systems with an instrument resolution of $0.02^{\circ}$. However, in the experiment, the fluctuations of the phase readings obtained from the lock-in amplifier amounts to $\pm 0.05^{\circ}$, which corresponds to an instrument working resolution of $0.1^{\circ}$, that in turn for a $\lambda_{2}=632-\mathrm{nm}$ wavelength, sets the surface profile measure- 


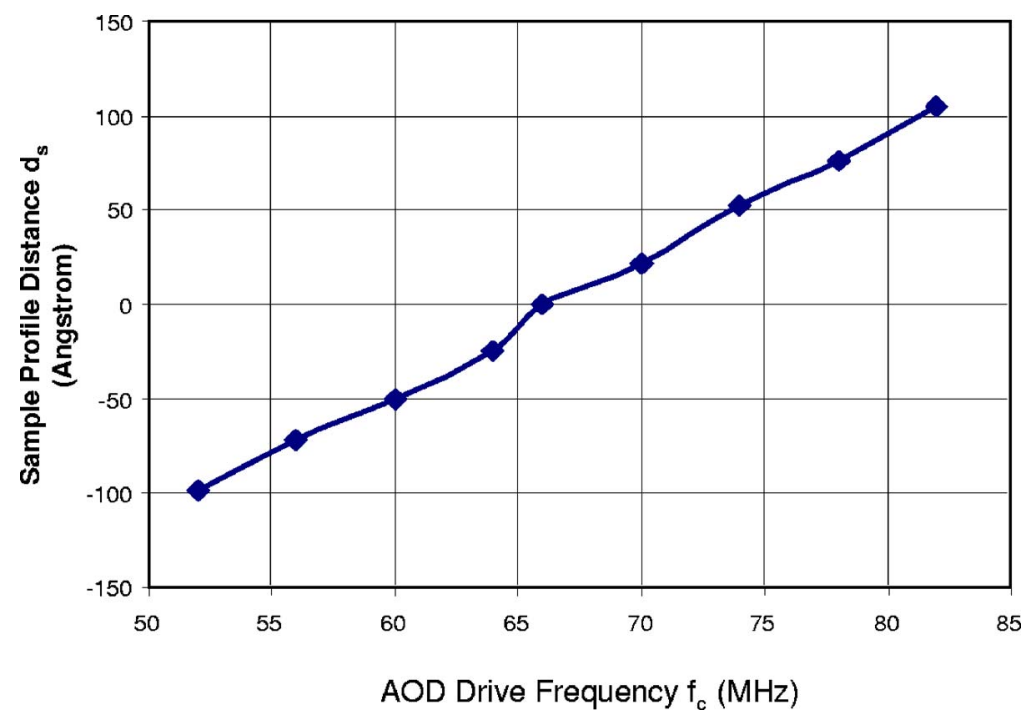

Fig. 3 Relative surface profile data of the reference mirror test plate obtained through scanning the AOD drive frequency $f_{c}$ from 50 to $85 \mathrm{MHz}$.

ment resolution to $0.9 \AA$ for the demonstrated interferometer. As the experimental fluctuations of $\pm 0.05^{\circ}$ are the fundamental noise limits of the experiment, it sets the noise level in terms of surface profile to be $\pm 0.45 \AA$. Therefore, the error bars on the experimental data can be set to $\pm 0.45 \AA$. The test mirror is scanned in one dimension over a distance of $490 \mu \mathrm{m}$ to get the relative profile variation as compared to the $70-\mathrm{MHz}$ reference position on the reference mirror (see Fig. 3). These Fig. 3 results for the test mirror surface quality demonstrate $\pm 100 \AA$ profile variations. As the sensing laser wavelength of $\lambda_{2}=632 \mathrm{~nm}$ is used in this experiment, reflective sample profile variation values up to $632 / 2=316 \mathrm{~nm}$ can be unambiguously and directly measured using the demonstrated interferometer. If advanced signal processing methods are deployed with reference hardware adjustments, the instrument measurement range can be increased.

\section{Hybrid-Multiplexed Acousto-Optic Interferometers}

The design proposed in Fig. 4 is a combination of the polarization-multiplexed scanning heterodyne interferometer, ${ }^{9,10}$ and the Fig. 1 wavelength-multiplexed scanning heterodyne interferometer. This hybrid design uses $N$ linearly polarized lasers with $N$ different wavelengths. The rotation of a half-wave plate (HWP) is used to control the intensity ratio between the $\mathrm{p}$ and $\mathrm{s}$ polarization components for each independent wavelength. If different ratios are required per wavelength to give full sensing flexibility, then each laser beam is accompanied by its own HWP placed before the grating. In this case, the input grating polarization dependent loss (PDL) effects must be taken into account. The $N$ wavelengths are incident at their respective Bragg angles on the input grating, producing an output diffracted beam where the $N$ colors are collinear and hence physically inline with each other. The AOD drive RF is chosen that sufficient Bragg diffraction is achieved simultaneously for all $N$ wavelengths. At the sensor chip plane, the AO diffraction process produces $2 N$ (+1-order) diffracted beams and $2 N$ DC beams. In effect, there are $2 N$ beam pairs, where one beam pair contains one +1 -order diffracted beam and its corresponding DC beam for the given wavelength and specific linear polarization. Of the $2 N$ beam pairs, $N$ beam pairs (one per wavelength) acting as the phase signal sensing signal generators are at the s-polarization while the corresponding $N$ beam pairs (one per wavelength) are the internal reference signal generators and are at the p-polarization. At the output of the interferometer, after double passage through the AOD, a PBS and an output grating are used to successfully generate the required phase and reference RF signals for the $N$ wavelengths. Note that depending on various system parameters such as wavelength values, Fourier transform cylindrical lens focal length, AOD device parameters, the $N$ diffracted beams from the $N$ wavelengths are spatially displaced in a linear array fashion. Thus, the proposed wavelengthpolarization-multiplexed scanning heterodyne interferometer indeed provides $N$ independent OPL measurements from the sensing zone of the $N$-element optical sensor chip. Moreover, because data are simultaneously taken at different wavelengths, the system forms an instantaneous interferometric spectroscopic tool useful in applications such as biomolecular sensors and chemical sensors.

One possible modification to the Fig. 4 system is shown in Fig. 5 and encompasses the replacement of the $N$ fixed wavelength lasers (or broadband laser) and their grating optics by a single tunable laser that, in time, generates multiple different wavelengths, one at a time, for timemultiplexed data measurements. In this case, the optics at the input and output ports are simpler as only two point photo-detectors are needed. In addition, the AOD RF drive can be changed to track the changing wavelengths, leading to possible wide band measurements over a given optical band. This modified interferometer can be called an internally referenced wavelength-polarization-time-multiplexed scanning heterodyne interferometer. Because laser tuning can be obtained at high speeds (e.g., ms to ns), high-speed, time-multiplexed data processing can lead to high- 


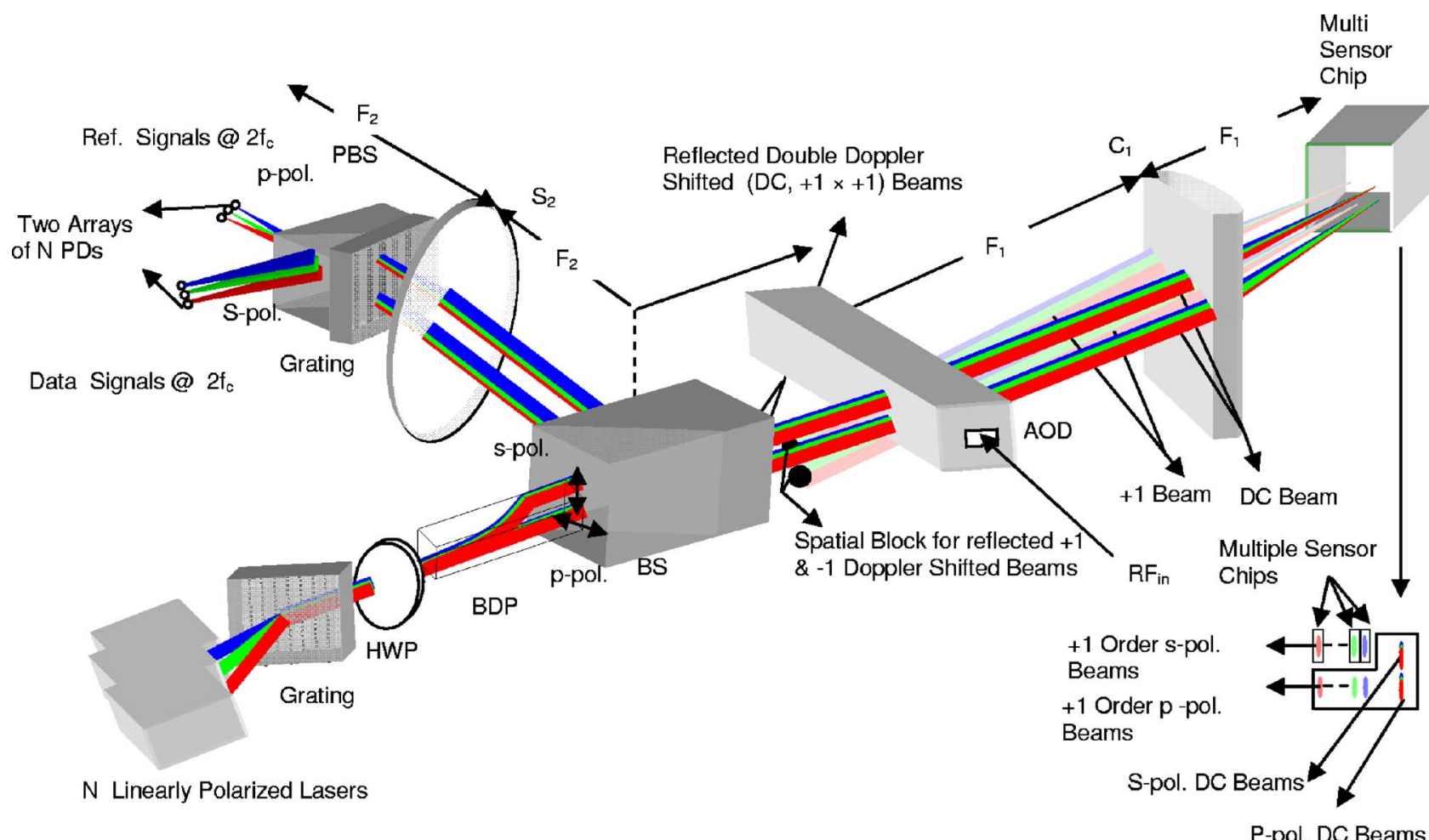

Fig. 4 Proposed design of the internally referenced hybrid wavelength-polarization multiplexed heterodyne interferometer working as a distributed spectral sensor.

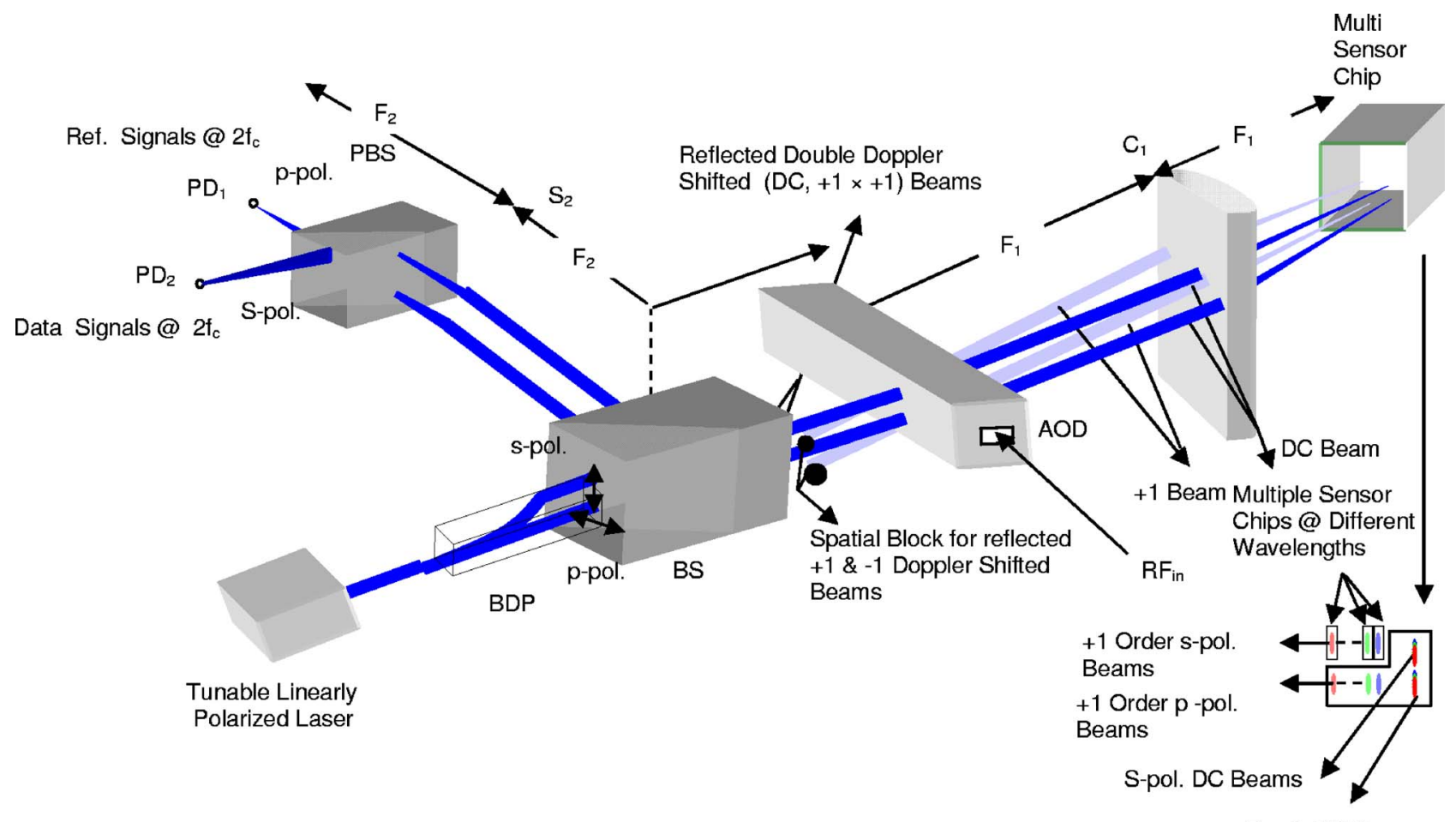

P-pol. DC Beams

Fig. 5 Proposed design of the internally referenced hybrid wavelength-polarization-time-multiplexed heterodyne interferometer working as a distributed spectral sensor. 
resolution distributed spectral sensing. In addition, compared to the Fig. 4 system, the Fig. 5 system avoids multiwavelength crosstalk as, at any given instant, only one wavelength is spatially active in the system. Nevertheless, this type of interferometer compared to Fig. 4 design does not take instantaneous sensing measurements that may be needed for capturing superfast transient effects in the sensing chip.

\section{Conclusion}

In conclusion, several internally referenced, multiplexed heterodyne interferometers have been proposed, including using wavelength multiplexing, wavelength-polarization multiplexing, and wavelength-polarization-time multiplexing. As a first example, the wavelength-multiplexed system is demonstrated as a 1D scanning interferometer for visible light-based surface profile sensing. Data from this instrument indeed show low noise Angstrom-level sensitivity. Future works relate to the implementations and testing of the other proposed hybrid interferometer designs.

\section{References}

1. P. Hariharan, "Interferometers," in Handbook of Optics 2, M. Bass, ed., McGraw-Hill, New York (1995).

2. E. W. Rogala and H. H. Barrett, "Phase-shifting interferometer/ ellipsometer capable of measuring the complex index of refraction and the surface profile of a test surface," J. Opt. Soc. Am. A 15,
$538-548$ (1998)

3. T. Shirai, T. H. Barnes, and T. G. Haskel, "Surface-profile measurement by means of a polarization Sagnac interferometer with paralleloptical feedback," Opt. Lett. 24, 297-299 (1999).

4. P. Pavlícek and G. Häusler, "White-light interferometer with dispersion: An accurate fiber-optic sensor for the measurement of distance," Appl. Opt. 44, 2978-2983 (2005).

5. D. Lin, X. Jiang, F. Xie, W. Zhang, L. Zhang, and I. Bennion, "High stability multiplexed fiber interferometer and its application on absolute displacement measurement and on-line surface metrology," Opt. Express 12, 5729-5734 (2004). http://www.opticsinfobase.org/ abstract.cfm? URI $=$ oe-12-23-5729.

6. N. A. Riza, "Scanning heterodyne optical interferometers," Rev. Sci. Instrum. 67, 2466-2476 (1996)

7. N. A. Riza and M. A. Arain, "Angstrom-range optical path-length measurement with a high-speed scanning heterodyne optical interferometer," Appl. Opt. 42, 2341-2345 (2003).

8. M. A. Arain and N. A. Riza, "Fiber coupled in-line heterodyne optical interferometer for minimally invasive sensing," J. Lightwave Technol. 23(8), 2449-2454 (2005).

9. N. A. Riza and M. A. Arain, "Angstrom sensitivity polarization multiplexed heterodyne acousto-optic interferometric sensor," in Proc. IEEE Sensors 2004 October 24-27, Vienna, Austria (Institute of Electrical and Electronic Engineers, New York, 2004).

10. M. A. Arain and N. A. Riza, "Precision polarization multiplexed heterodyne acousto-optic interferometric sensor," Opt. Eng. 44(5), 054401 (2005)

11. M. A. Arain and N. A. Riza, "Self-calibrating wavelength multiplexed heterodyne interferometer for angstrom precision," Proc. SPIE 5814, 140-143 (2005). Enabling Photonics Technologies for Defense, Security, and Aerospace Applications; A. R. Pirich, M. J. Hayduk, E. J. Donkor, and P. J. Delfyett Jr., eds. (2005).

Biographies and photographs of the authors are not available. 